\title{
Annihilation characteristics of positrons in a polymer containing silver nanoparticles
}

\author{
M. Mukherjee* and D. Chakravorty ${ }^{\dagger}$ \\ Indian Association for the Cultivation of Science, Jadavpur, Calcutta 700 032, India \\ P. M. G. Nambissan \\ Saha Institute of Nuclear Physics, 1/AF Bidhannagar, Calcutta 700 064, India \\ (Received 16 July 1996; revised manuscript received 14 August 1997)
}

\begin{abstract}
Composites of silver particles of diameters in the range 16.4-33.3 $\mathrm{nm}$ and polyacrylamide were prepared by a chemical method. Positron lifetime and Doppler broadening measurements of these samples were carried out. The positron lifetime spectra of all the samples could be decomposed into three components having lifetimes around 200, 500, and $1800 \mathrm{ps.} \mathrm{These} \mathrm{are} \mathrm{believed} \mathrm{to} \mathrm{arise} \mathrm{due} \mathrm{to} \mathrm{vacancy} \mathrm{clusters} \mathrm{on} \mathrm{the} \mathrm{grain} \mathrm{surfaces,} \mathrm{the}$ open spaces between the grain surface and the surrounding polymer layer and the annihilation of orthopositronium at the free-volume defects, respectively. The lifetime of positrons trapped at the grain surface defects and the grain-polymer interface is found to decrease as the grain size is increased. Doppler broadening measurements were carried out from $13 \mathrm{~K}$ to $300 \mathrm{~K}$ on a silver-polyacrylamide nanocomposite containing silver particles of diameters in the range $2-20 \mathrm{~nm}$. The line-shape parameter $S$ is decomposed by a mixture rule to obtain the contribution of electrons from the nanoparticles. This shows a sharp increase at around $80 \mathrm{~K}$ that is adduced as evidence for the splitting of the electron energy levels in the nanosized silver particles leading to a semiconductorlike behavior. [S0163-1829(98)03102-6]
\end{abstract}

\section{INTRODUCTION}

The structure and stability of nanocrystalline materials are nowadays being widely investigated due to the interest in their unusual physical properties. ${ }^{1,2}$ The disorder in the arrangement of atoms at the interfaces of grains or particles of size of the order of a few tens of nanometers have to be taken into account for explaining the anomalous features exhibited by these materials. Several experimental methods have been adopted to characterize the diffuse vacancy clusters formed at the grain interfaces. ${ }^{3,4}$ Positron annihilation spectroscopy is most suitable for the investigation of these interfacial defects due to high sensitivity of the technique to defect-related processes and the ability of thermalized positrons to diffuse out and annihilate at the surfaces of grains smaller than their thermal diffusion wavelength. ${ }^{4,5}$

Nanocrystalline materials are generally prepared by the conventional metal-evaporation and condensation method. ${ }^{6,7}$ In this work, we have been able to grow nanometer-sized silver particles inside a polyacrylamide polymer gel by chemical methods. This method of production of nanoparticles has certain advantages. It provides means to effectively control the growth process by suitably adjusting the experimental parameters like temperature and time of heat treatment, material concentration, and choice of suitable chemical reagents. Since the majority of atoms reside on the grain boundaries, these particles yield to a great degree of compaction and hence small pellets of definite shape and size can be prepared by applying mechanical pressure, without introducing dislocations in the crystal structure. The presence of silver nanoparticles inside the polymer was confirmed both by transmission electron microscopy and x-ray diffraction and their sizes were estimated. Both positron lifetime and Doppler broadening measurements were carried out to characterize samples containing nanoparticles of varying sizes and to monitor the processes during isochronal and isothermal annealing.

With the synthesis of nanometer-sized metal particles becoming a reality, it is possible now to investigate the mechanism leading to such remarkable deviation from the bulk properties of materials using various spectroscopic probes. A particle of radius about $2 \mathrm{~nm}$ may consist of clusters of a few hundred atoms. The metallic character of the particle has been reported to persist even up to that size. ${ }^{8}$ For particles of smaller sizes, one would expect a metal-to-insulator transition to occur. ${ }^{9}$ For the energy levels of such a small particle will then be discrete, as pointed out by Wood and Ashcroft ${ }^{9}$ and the energy gap between the lowest unoccupied level and the highest occupied level will be such that a transition of the above type can be observed at a particular temperature. Section III E of this paper in fact deals with the results of an investigation to elucidate this aspect on a polymer nanocomposite consisting of silver nanoparticles and reports significant anomalies at temperatures at which phase transition was expected from simple quantum-mechanical considerations.

\section{EXPERIMENTAL PROCEDURE}

\section{A. Sample preparation}

For preparing the polymer-silver nanocomposites $4 \mathrm{~g}$ of polyacrylamide (supplied by BDH Chemicals, UK) with molecular weight greater than $5 \times 10^{6}$ was dissolved in $450 \mathrm{~cm}^{3}$ of distilled water (solution denoted by $A$ ). Another solution was prepared by dissolving $0.5 \mathrm{~g} \mathrm{AgNO}_{3}$ in distilled water. To this sodium hydroxide solution was added until complete precipitation of $\mathrm{Ag}_{2} \mathrm{O}$ occured. After washing the precipitate in distilled water it was dissolved in $20 \mathrm{~cm}^{3}$ dilute $\mathrm{NH}_{4} \mathrm{OH}$ (solution denoted by $B$ ). $160 \mathrm{~cm}^{3}$ of solution $A$ was then mixed with solution $B$ and excess formic acid $(p H \sim 2)$ was added for reduction of $\mathrm{Ag}_{2} \mathrm{O}$ to metallic silver. Ethyl alcohol 
TABLE I. Average grain size and the bulk density of the various polymer-silver nanocomposite samples.

\begin{tabular}{|c|c|c|c|}
\hline Sample & $\begin{array}{c}\text { Amount of } \mathrm{AgNO}_{3} \\
\text { in precursor } \\
\text { solution } \\
B(\mathrm{~g})\end{array}$ & $\begin{array}{l}\text { Grain size } \\
\quad(\mathrm{nm})\end{array}$ & $\begin{array}{c}\text { Density } \\
\left(\mathrm{g} / \mathrm{cm}^{-3}\right)\end{array}$ \\
\hline Pure polymer & & & 1.12 \\
\hline 1 & 0.5 & 16.4 & 1.15 \\
\hline 2 & 1.0 & 19.5 & 1.16 \\
\hline 3 & 2.0 & 22.0 & 1.38 \\
\hline 4 & 16.0 & 25.8 & 3.33 \\
\hline 5 & 8.0 & 29.7 & 3.01 \\
\hline 6 & 16.0 & 33.3 & 5.17 \\
\hline
\end{tabular}

was added to precipitate out the composite from water. The powder obtained was packed tightly in a perspex mould and dried in an oven at $323 \mathrm{~K}$ for $24 \mathrm{~h}$. The powder in the mould was compacted by applying a pressure of $3 \mathrm{GPa}$ to form pellets of $1-\mathrm{cm}$ diameter and 3-mm thickness. For preparing samples with different diameters of the silver nanoparticles the procedure was identical to that described above except that at the preparation stage of solution $B$ the amount of $\mathrm{AgNO}_{3}$ taken was varied from 0.5 to $16 \mathrm{~g}$. The amounts taken in the case of different samples are given in Table I. For sample 6 the synthesis was carried out at a temperature of $363 \mathrm{~K}$. Polyacrylamide pellets were prepared by following the procedure outlined as above without the addition of $\mathrm{Ag}_{2} \mathrm{O}$.

\section{B. Transmission electron microscopy and x-ray diffraction}

The microstructure of the various specimens were studied using a JEM 200 CX transmission electron microscope operated at $160 \mathrm{kV}$ at the Regional Sophisticated Instrumentation Centre, Bose Institute, Calcutta. The silver phase was identified by the electron-diffraction pattern. The x-raydiffraction patterns were taken using a Phillips Analytical PW1877 automated powder diffractometer employing $\mathrm{CuK}_{\alpha}$ radiation. The sizes of the silver nanoparticles were estimated from the x-ray-diffraction data using the Scherrer equation. $^{10}$

\section{Positron lifetime and Doppler broadening measurements}

The thickness of the specimens was so chosen to ensure that all positrons emitted by the source ultimately annihilated within the specimens only. A thin deposition of ${ }^{22} \mathrm{NaCl}(\sim 1 \mu \mathrm{Ci})$ over an area of about $2 \mathrm{~mm}$ on a thin $\left(2 \mathrm{mg} / \mathrm{cm}^{-2}\right)$ nickel foil and covered by an identical foil was used as the source of positrons and sandwiched between two identical pellets. For Doppler broadening measurements, an HPGe detector of $1.14 \mathrm{keV}$ resolution [full width at half maximum (FWHM)] at $514 \mathrm{keV} \gamma$ ray from ${ }^{85} \mathrm{Sr}$ was used. About a million counts were collected under each Doppler broadened $511 \mathrm{keV} \gamma$-ray spectrum. The positron lifetime spectra were recorded using a fast-fast coincidence setup with a time resolution (FWHM) of $260 \mathrm{ps}$ for ${ }^{60} \mathrm{Co} \gamma$ rays accepted under experimental windows. During the data acquisition, the specimens-source sandwich was kept inside a glass tube and was continuously evacuated to avoid the absorption of air and moisture by the polymer samples. Hence the two scintillation detectors of the coincidence spectrometer had to be pulled apart to about $20 \mathrm{~mm}$ distance between them. Invariably this resulted in a drastic reduction in the count rate but we purposely avoided using a stronger source, keeping in mind the possible effects of positron irradiation on the polymer samples. ${ }^{11,12}$ About 0.4 million counts were collected under each lifetime spectrum. The Doppler broadened line shape of the positron annihilation $\gamma$-ray spectrum was parametrized by the ratio of the area falling under that segment of the spectrum covering the energy range from 510.4 to $511.6 \mathrm{keV}$ to the area under the total spectrum covering from 503.3 to $518.7 \mathrm{keV}$. This parameter was denoted by $S$. The positron lifetime spectra were analyzed using RESOLUTION and POSITRONFIT ${ }^{13}$ until the multicomponent fit of all the spectra yielded variance of fit between 0.95 and 1.20 .

Measurements were carried out on pure polyacrylamide samples and then six other samples, each containing silver nanoparticles of a different average size. Besides, additional measurements were conducted on samples containing silver nanoparticles of a given mean size but after isochronally (30 min) annealing at different temperatures. A fresh set of specimens were used for each isochronal annealing, so as to rule out the effects of positron and $\gamma$ irradiation. The specimens thus annealed at $473 \mathrm{~K}$ were then isothermally annealed and the changes were monitored by conducting similar measurements after different intervals of annealing. The effects of annealing on the pure polyacrylamide polymer sample were also studied. All the measurements were performed at room temperature. To avoid the bulk expansion of the polymer samples during the heat treatment, all the annealings were conducted by enclosing the pellets into a rigid stainless-steel mould. (The details of special experimental arrangements used for the low-temperature measurements are given in Sec. III E.)

\section{RESULTS AND DISCUSSION}

\section{A. Microstructure}

In Table I the sizes of silver particles as estimated from the X-ray powder pattern are summarized. Figure 1(a) is a typical transmission electron micrograph obtained for sample 4. Figure 1(b) is the corresponding electron-diffraction pattern. In Table II the interplanar spacings $d_{h k l}$ estimated from the ring diameters of Fig. 1(b) are compared with standard American Society for Testing and Materials (ASTM) data. It is evident that the particles comprise of metallic silver. We have also analyzed the particle size distribution in Fig. 1(a) by a log-normal distribution function. ${ }^{14}$ The median diameter $\bar{x}$ and the geometric standard deviations $\sigma$ as extracted from such an analysis are found to be 30.4 and $1.5 \mathrm{~nm}$, respectively. It is evident that $\mathrm{x}$-ray analysis gives a lower value $(25.8 \mathrm{~nm})$ for the average particle size. This is believed to arise due to the fact that $\mathrm{x}$ rays probe the smallest particles, whereas by transmission electron microscopy the agglomerated particles are better identified.

\section{B. Positron lifetimes in the polymer sample}

The positron lifetime spectra of all the samples were best resolved into three components, designated as $\tau_{1}, \tau_{2}$, and $\tau_{3}$ 


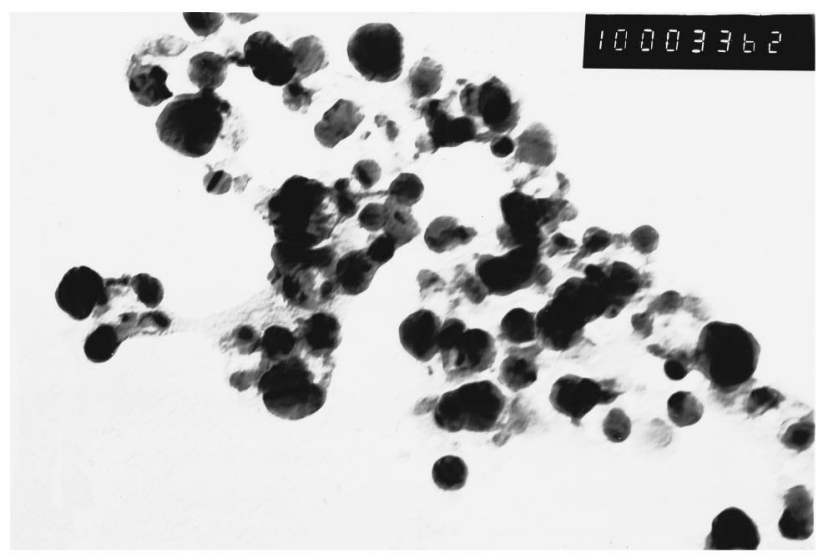

(a)

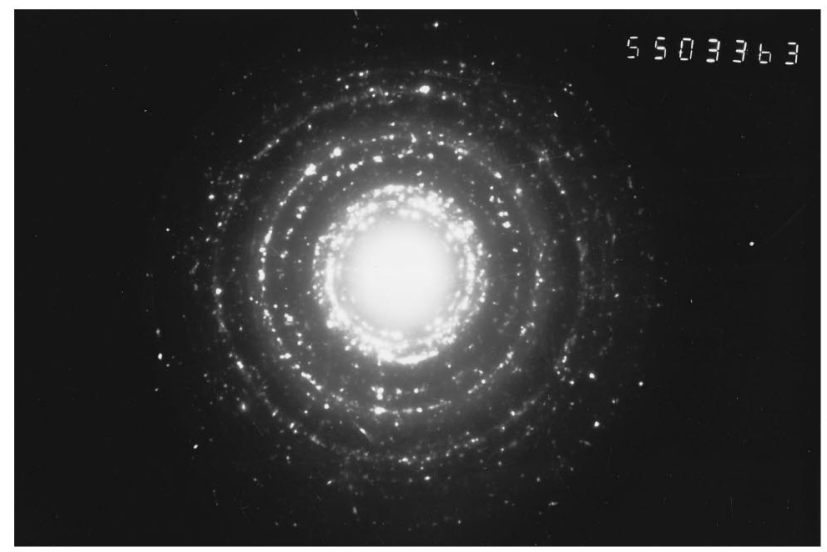

(b)

FIG. 1. Transmission electron micrograph for sample 4. $\times 190,000$. (b) Electron diffraction pattern for Fig. 1(a).

in ascending order of their values and with relative intensities $I_{1}, I_{2}$, and $I_{3}$. From the spectrum of the pure polyacrylamide sample, we obtained $\tau_{1}=139 \mathrm{ps}, \tau_{2}=370 \mathrm{ps}$, and $\tau_{3}=1.62 \mathrm{~ns}$ with the respective intensities of $30.0 \%, 51.3 \%$, and $18.7 \%$. For the physical interpretation of these results, we note that the value of $\tau_{1}$ is slightly higher than the lifetime associated with the self-decay of parapositronium (p-Ps) atoms in polymers, which is known as 125 ps. The explanation for the origin of $\tau_{2}$ is deferred to a later section. The longest lifetime $\tau_{3}$ is the result of the pickoff annihilation of orthopositronium (o-Ps) atoms formed in the free-volume defects in the amorphous polymer material. The rate of pickoff, i.e., the process in which the positron in the o-Ps atom is

TABLE II. Comparison of interplanar spacings $d_{h k l}$ obtained from electron diffraction with standard ASTM data for silver metal in sample no. 4.

\begin{tabular}{cc}
\hline \hline $\begin{array}{c}\text { Observed } \\
(\mathrm{nm})\end{array}$ & $\begin{array}{c}\text { ASTM } \\
(\mathrm{nm})\end{array}$ \\
\hline 0.236 & 0.2359 \\
0.204 & 0.2044 \\
0.144 & 0.1445 \\
0.123 & 0.1231 \\
0.094 & 0.09375 \\
\hline \hline
\end{tabular}

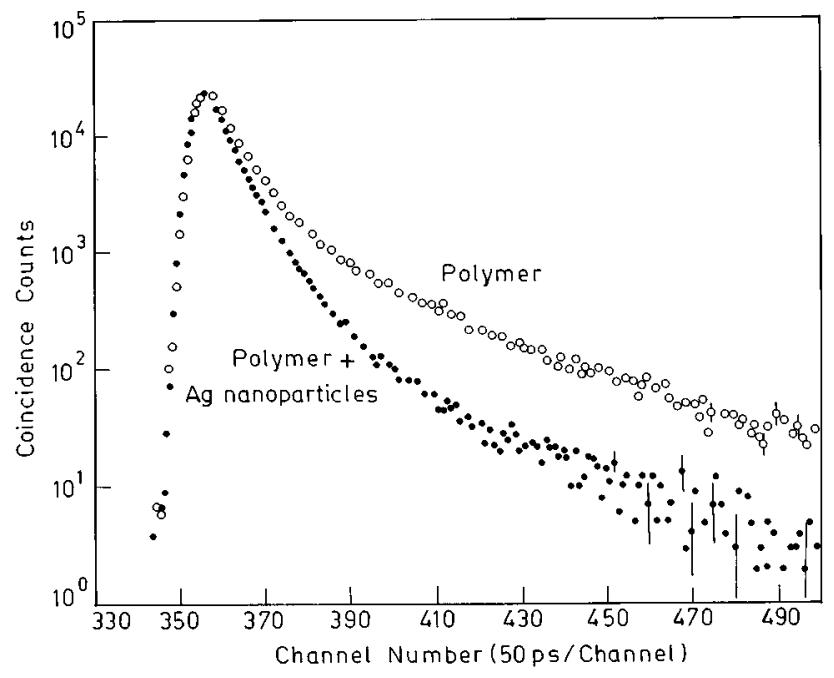

FIG. 2. Positron lifetime spectra of (a) pure polyacrylamide polymer and (b) the polymer containing silver nanoparticles (sample 6), showing the changes in the annihilation characteristics due to the presence of the nanoparticles.

picked by an electron with antiparallel spin from the surroundings, thus resulting in faster annihilation, depends on the structure and contamination of the internal surfaces of the free-volume defects. ${ }^{2}$

The lifetime $\tau_{1}$ is an admixture of the p-Ps self-decay component (125 ps) and the lifetime of positrons annihilating in the bulk polymer sample. A scheme of analysis used by Uedono et al. $^{15}$ assumes the intensity of the p-Ps lifetime component to be $I_{3} / 3$ as the ratio of the formation probability of o-Ps to that of p-Ps is 3:1. From the lifetime (125 ps) and intensity $\left(I_{3} / 3\right)$ of the p-Ps component and from the measured values of $\tau_{1}$ and $I_{1}$, the lifetime of positrons annihilating in the bulk polymer can be estimated as $142 \mathrm{ps}$. On the other hand, the measured value of $\tau_{3}$ can be used to estimate the size of the free-volume defects using the semiempirical relation $^{16,17,18}$

$$
\tau_{3}=0.5\left[1-\left(R / R_{0}\right)+\left(\frac{1}{2}\right) \sin \left(2 \pi R / R_{0}\right)\right]^{-1},
$$

where $\tau_{3}$ is in ns and the free-volume radius $R$ is in $\AA$. Also $R_{0}=R+\Delta R$ where $\Delta R$ is the fitted empirical electron layer thickness (=1.66 $\AA$ ). Also calculated was the free-volume fraction $f$, which is expressed as $f=A V_{f} I_{3}$ where $V_{f}$ is the size of the free volumes and $A$ is a constant. The significance of these values are discussed later.

\section{Positron annihilation in the polymer containing silver nanoparticles}

The positron lifetime spectra of the polymer containing silver nanoparticles appear remarkably different from that of the pure polymer (Fig. 2). The resolved lifetimes and their intensities along with the values of the $S$ parameter obtained from the Doppler broadened line shape are shown in Table III. The values of $\tau_{1}$ of these spectra were significantly larger than that in the case of the polymer sample. It was therefore obvious that, in addition to the p-Ps lifetime and the lifetime of positrons annihilating in the bulk of the polymer, a third component is being admixed in the resultant $\tau_{1}$ obtained in 
TABLE III. Positron lifetimes, intensities, and the $S$ parameter for the various polymer-silver nanocomposite samples. The values for the pure polyacrylamide polymer sample are also given for comparison. Error in $S$ is \pm 0.0008 .

\begin{tabular}{lccccccc}
\hline \hline Sample & $\tau_{1}(\mathrm{ps})$ & $\tau_{2}(\mathrm{ps})$ & $\tau_{3}(\mathrm{ps})$ & $I_{1}(\%)$ & $I_{2}(\%)$ & $I_{3}(\%)$ & $S$ \\
\hline Polymer & $139 \pm 9$ & $370 \pm 11$ & $1623 \pm 19$ & $30.1 \pm 2.7$ & $51.3 \pm 2.4$ & $18.7 \pm 0.4$ & 0.4030 \\
1 & $205 \pm 7$ & $508 \pm 30$ & $1875 \pm 38$ & $53.2 \pm 3.1$ & $31.5 \pm 2.7$ & $15.3 \pm 0.6$ & 0.4016 \\
2 & $195 \pm 6$ & $467 \pm 24$ & $1785 \pm 28$ & $51.5 \pm 3.0$ & $33.0 \pm 2.7$ & $15.5 \pm 0.5$ & 0.4014 \\
3 & $181 \pm 7$ & $424 \pm 19$ & $1694 \pm 25$ & $46.3 \pm 3.3$ & $39.1 \pm 3.0$ & $14.6 \pm 0.4$ & 0.3996 \\
4 & $188 \pm 7$ & $386 \pm 17$ & $1679 \pm 54$ & $57.5 \pm 4.4$ & $38.5 \pm 4.3$ & $4.0 \pm 0.2$ & 0.3863 \\
5 & $157 \pm 8$ & $349 \pm 12$ & $1504 \pm 36$ & $41.1 \pm 4.0$ & $52.6 \pm 3.9$ & $6.3 \pm 0.3$ & 0.3898 \\
6 & $169 \pm 6$ & $358 \pm 12$ & $1753 \pm 59$ & $56.3 \pm 3.7$ & $40.7 \pm 3.6$ & $3.0 \pm 0.2$ & 0.3836 \\
\hline \hline
\end{tabular}

the analysis. Our efforts to separate this component from $\tau_{1}$ by fitting four components in the multiexponential decaying spectra did not yield satisfactory results and the spectra were best fitted for three components only. Hence, to get some insight into the physics of this aspect, we assumed that the lifetime and intensity of the positrons annihilating in the bulk of the polymer are necessarily the same in both the pure polymer and in the polymer-silver nanocomposite. Decomposing thus $\tau_{1}$ of the latter into three components in terms of the known values of the p-Ps lifetime (125 ps), its intensity $\left(I_{3} / 3\right)$, the bulk polymer contribution (142 ps; $\left.23.9 \%\right)$, and the measured values of $\tau_{1}$ and $I_{1}$, we estimated the third admixed lifetime $\tau_{1}^{\prime}$ and its intensity $I_{1}^{\prime}$. The values of this component ranged between 177 and 278 ps. Figure 3 shows the systematic decrease of $\tau_{1}^{\prime}$ with increasing grain size. Such lifetimes, which are in close agreement with $\tau_{1}^{\prime}$ obtained in the present work, have also been reported earlier by Qin et al. in their studies on nanocrystalline silver grain

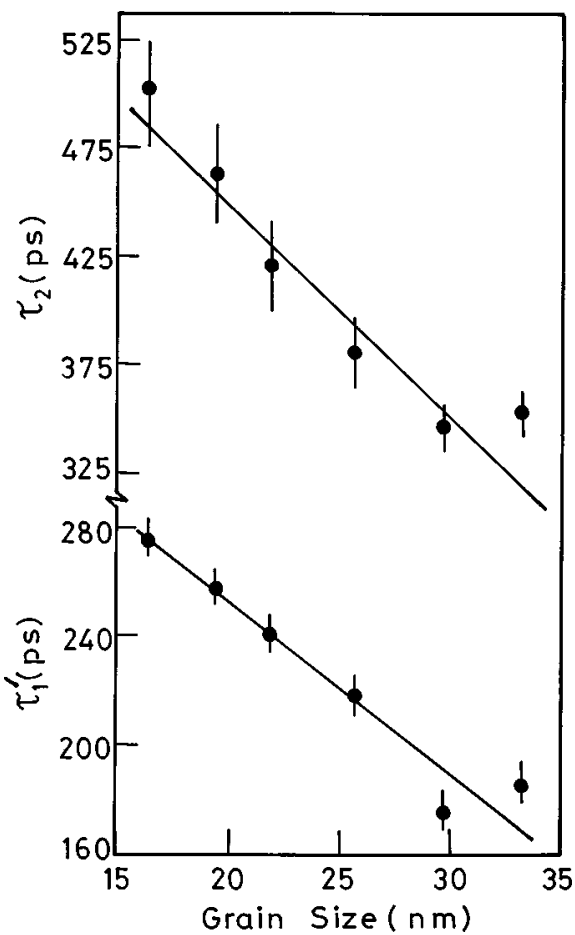

FIG. 3. Estimated lifetimes of positrons annihilating at the diffuse vacancy clusters on the grain surface $\left(\tau_{1}^{\prime}\right)$ and at the grainpolymer interface $\left(\tau_{2}\right)$ vs the average grain size. interfaces. ${ }^{7}$ The origin of this component is attributed to the diffuse vacancy clusters on the surfaces of the nanometersized silver grains in the polymer. Positrons captured within the silver grains can thermally diffuse out to the surfaces prior to annihilation as the grain size is smaller than the thermal diffusion wavelength $(\sim 110 \mathrm{~nm})$ of positrons in silver. ${ }^{19}$ On the grain surfaces, they are trapped by the vacancy clusters, resulting in a lifetime characteristic of the nature of the grain interface and the interfacial defect structures. Tong et al. have also reported on the decrease of positron lifetime with increasing grain size and attributed it to the reduction in trap volume available on the interfaces of larger grains. ${ }^{20}$

It appears rather interesting to note a striking difference between the value of the intermediate lifetime $\tau_{2}$ in the pure polymer $(=370 \mathrm{ps})$ and in the polymer-silver nanocomposites $(=358-508 \mathrm{ps})$. To explore the origin of this lifetime, we turn to Eq. (1) where the measured lifetime of o-Ps atoms bears a correlation with the dimension of the free-volume cavity in which they are formed. Uedono et al. ${ }^{15}$ pointed out that o-Ps formation might not be favored if the size of the free-volume cavity was less than $0.1 \mathrm{~nm}$. Equation (1) would then imply a lifetime of at least 680 ps for an o-Ps atom decaying via pickoff. Since the values of $\tau_{2}$ in both the polymer and the polymer-silver nanocomposites are less than this limit, this component can be attributed to positrons trapped in the open spaces of dimensions smaller than $0.1 \mathrm{~nm}$. While this interpretation seems to be plausible for the pure polymer sample, it does not fully describe the larger values of $\tau_{2}(500$ ps) in the polymer-silver nanocomposites. It appears that another lifetime component has merged with the lifetime of positrons trapped in the open spaces described above. To understand the origin of this second component admixed in $\tau_{2}$, we note that the silver nanoparticles are actually grown as embedded in a polymer matrix. The open spaces between the grain surface and the innermost polymer layer surrounding it can act as the most stable state of the positrons reaching at this interface. ${ }^{21}$ An analogy can be drawn between the situation described here and the case of an inert gas bubble formed in a metal matrix. ${ }^{22}$ In such a case where positrons can be trapped by a bubble, the most stable state of the positron was found localized at the metal-gas interface. ${ }^{23}$ Molecular dynamical simulations of the atomic structure of gas bubbles and the positron states at the interface provided the most realistic interpretation of the experimentally measured positron lifetimes in a number of metal-inert gas 
TABLE IV. Positron lifetimes, intensities, and $S$ parameter for the annealed polymer-silver nanocomposite samples.

\begin{tabular}{lccccccc}
\hline \hline Sample & $\tau_{1}(\mathrm{ps})$ & $\tau_{2}(\mathrm{ps})$ & $\tau_{3}(\mathrm{ps})$ & $I_{1}(\%)$ & $I_{2}(\%)$ & $I_{3}(\%)$ & $S$ \\
\hline As-prepared & $205 \pm 7$ & $508 \pm 30$ & $1875 \pm 38$ & $53.2 \pm 3.1$ & $31.5 \pm 2.7$ & $15.3 \pm 0.6$ & 0.4016 \\
$373 \mathrm{~K}$ & $222 \pm 5$ & $591 \pm 33$ & $1964 \pm 42$ & $59.6 \pm 2.1$ & $26.5 \pm 1.7$ & $13.9 \pm 0.6$ & 0.4042 \\
$398 \mathrm{~K}$ & $194 \pm 6$ & $517 \pm 25$ & $1952 \pm 41$ & $51.9 \pm 2.6$ & $34.2 \pm 2.2$ & $13.9 \pm 0.6$ & 0.4045 \\
$448 \mathrm{~K}$ & $191 \pm 6$ & $485 \pm 22$ & $1800 \pm 29$ & $49.8 \pm 2.5$ & $34.3 \pm 2.2$ & $15.9 \pm 0.5$ & 0.4046 \\
$473 \mathrm{~K}$ & $199 \pm 6$ & $495 \pm 26$ & $1798 \pm 30$ & $52.9 \pm 2.7$ & $31.4 \pm 2.4$ & $15.7 \pm 0.5$ & 0.4034 \\
$498 \mathrm{~K}$ & $182 \pm 7$ & $430 \pm 18$ & $1664 \pm 21$ & $45.4 \pm 3.0$ & $37.6 \pm 2.7$ & $17.0 \pm 0.4$ & 0.4029 \\
\hline \hline
\end{tabular}

bubble systems. ${ }^{23}$ We presume that an identical situation can be realized for positrons trapped at the grain-polymer interface and give a characteristically longer lifetime. This component together with the lifetime of positrons trapped in the open spaces of dimensions smaller than $0.1 \mathrm{~mm}$ in the polymer matrix can then explain the occurrence of $\tau_{2}$ in the spectra of the polymer-silver nanocomposites. Since the distribution of the fractions of positrons annihilating in the open spaces and the grain-polymer interfaces is not clear, it has not been possible to estimate the lifetime of positrons trapped at the interfaces. However, once again the values of $\tau_{2}$ show a systematic reduction with increasing grain size (Fig. 3), which vindicates the validity of the interpretation given above.

\section{Effects of annealing}

In order to further elucidate the kinetics of formation and growth of silver nanoparticles in the polymer medium, isothermal and isochronal annealing were performed on one of the samples. Here the sample with initial mean grain size of $16.4 \mathrm{~nm}$ had been chosen. Because of the possible irradiation effects by positrons, a fresh pair of specimens was used for each isochronal annealing and measurements. The isochronal annealing of the pure polymer was also performed as a reference. But this did not result in any noticeable changes in the shorter lifetimes $\tau_{1}$ and $\tau_{2}$ or in their intensities. The only change observed was a reduction of the third component $\tau_{3}$ from 1.62 to 1.45 ns. From Eq. (1), this change can

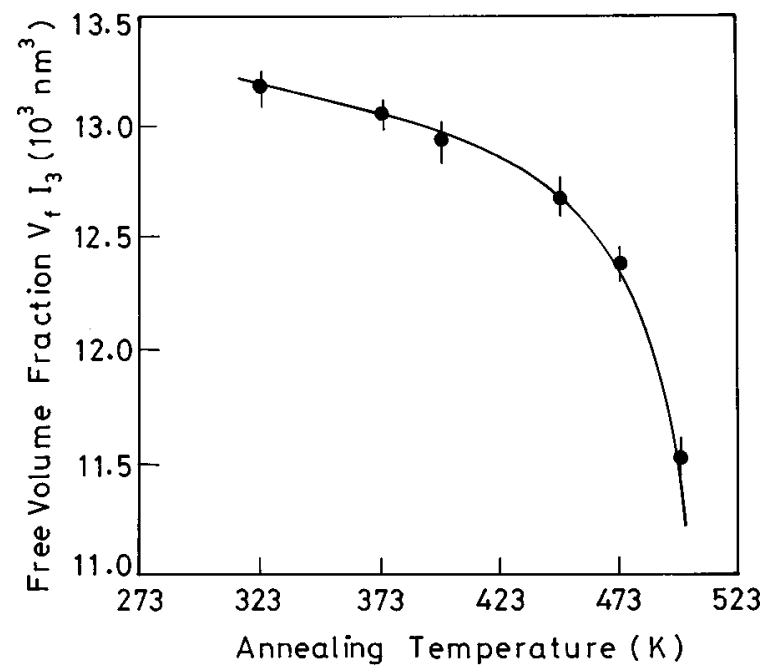

FIG. 4. The estimated free-volume fraction as a function of annealing temperature of sample 1 . be assigned to a reduction of the free-volume radius from 0.25 to $0.23 \mathrm{~nm}$. Since the polymer was annealed in a tightly enclosed stainless-steel mould, its expansion in volume due to temperature was prevented. The bulk polymer therefore has to expand by shrinking the free-volume cavities. The effects of annealing on the growth of grains and the evolution of the defects in the polymer-silver nanocomposites are reflected as changes in the positron lifetime parameters, as shown in Table IV. The temperature stages indicating the important annealing features are in general consistent in both lifetime and $S$ parameter measurements. A careful study of the lifetimes and the $S$ parameter of Table IV will indicate the following annealing stages.

From the as-prepared state to the state after the annealing at $373 \mathrm{~K}$, all the positron lifetime components increase. The intensities $I_{2}$ and $I_{3}$ decrease as $I_{1}$ shows a small rise in its magnitude. The $S$ parameter clearly depicts a substantial increase due to the annealing at $373 \mathrm{~K}$.

All these trends are just reversed during the annealing at subsequent temperatures where a gradual change in the annihilation parameters is observed.

The explanation given for the changes in $\tau_{3}$ (and $I_{3}$ ) in the case of the polymer sample also holds good for the observed gradual decrease of $\tau_{3}$ of the spectra of the polymersilver nanocomposite during the isochronal annealing. In fact, the free-volume fraction, estimated as described in Sec. III B, exhibits a gradual reduction with annealing temperature, as shown in Fig. 4. The variation of the other two lifetime parameters $\tau_{1}$ and $\tau_{2}$, and their intensities $I_{1}$ and $I_{2}$, describes the effects of annealing on the nanoparticles. The mean particle sizes, as estimated from the x-ray-diffraction data of the annealed samples, are given in Table V. The reduced particle size in the sample annealed at $373 \mathrm{~K}$ is reflected as a slight enhancement in the positron lifetimes, as explained in Sec. III C. Compared to these values of $\tau_{1}$ and $\tau_{2}$, the values at higher annealing temperatures gradually fall

TABLE V. Average grain size in sample 1 after annealing at various temperatures.

\begin{tabular}{cc}
\hline \hline Temperature $(\mathrm{K})$ & Grain size $(\mathrm{nm})$ \\
\hline As-prepared & 16.4 \\
373 & 12.2 \\
398 & 15.1 \\
448 & 13.1 \\
473 & 16.6 \\
498 & 16.9 \\
\hline \hline
\end{tabular}




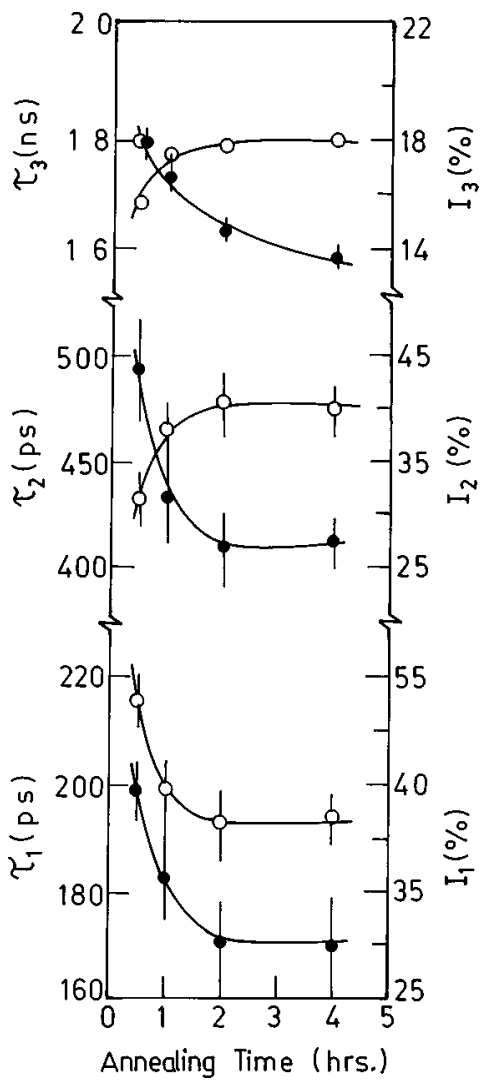

FIG. 5. The variation in the measured positron lifetimes (closed circles) and intensities (open circles) due to isothermal annealing of sample 1 at $473 \mathrm{~K}$.

and is supported by a general trend of increasing particle sizes, as shown in Tables IV and V. The absence of a welldefined nature for the relation between $\tau_{1}^{\prime}$ (estimated as described earlier in Sec. III B) or $\tau_{2}$ with mean grain size here (unlike in Fig. 3) may be due to the extremely narrow range of size distribution of the particles in the whole lot of the heat-treated samples. The intensities $I_{1}$ and $I_{2}$ are more or less the same in all the heat-treated samples implying that the isochronal annealing did not necessarily change the interfacial diffuse vacancy cluster concentration or the trap volume at the grain-polymer interface.

On the other hand, the isothermal annealing at $473 \mathrm{~K}$ resulted in significant changes as shown in Fig. 5. The mean grain size $(16.6 \mathrm{~nm})$ did not change even after $4 \mathrm{~h}$ of annealing. Hence the observed variations in the positron lifetime parameters should be understood in terms of the annealing of defects or the interdiffusion of the softened polymer and the dangling surface atoms of the nanocrystalline grains.

\section{E. Low-temperature measurements: A metal-to-semiconductorlike transition?}

In one of the silver-polymer nanocomposite sample prepared (not included in the experiments discussed in the previous sections), silver nanoparticles of extremely low size could be detected from transmission electron microscopy (TEM). The histogram of silver nanoparticle size distribution in this sample obtained from the TEM picture is shown in Fig. 6(a). It shows a significant number of nanoparticles even of diameters below $5 \mathrm{~nm}$. Such a composite is ideal for seek-

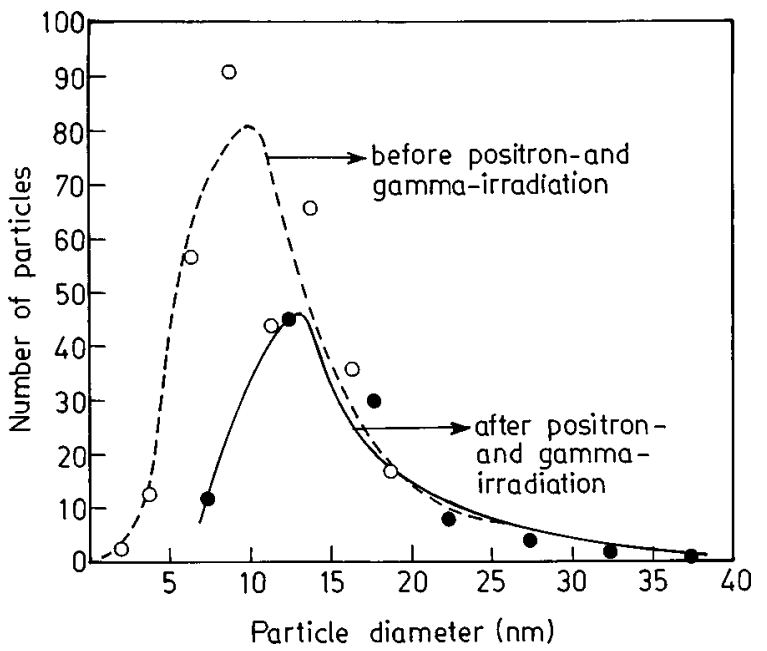

FIG. 6. The histogram of silver nanoparticle size distribution in the nanocomposite from the TEM picture taken (a) before and (b) after the low-temperature experiments.

ing to identify the splitting of the one-electron energy levels leading to the semiconductorlike transition predicted by the Wood-Aschroft theory. ${ }^{9}$ We conducted Doppler broadening measurements on this sample and the results are discussed.

A ${ }^{22} \mathrm{Na}$ positron source of approximate strength $10 \mu \mathrm{Ci}$ was sandwiched inbetween two such pellets and then mounted onto the sample holder attached to the cold head of a Leybold refrigerator cryostat. During the experiments, the temperature of the cold head was set and held stable (within $0.3 \mathrm{~K}$ ) by a temperature controller, LTC 60 . The actual temperature of the sample at each of the selected temperatures was accurately measured by mounting another DT450 temperature sensor very close to the sample. Initially both positron lifetime and Doppler broadening measurements were carried out at room temperature. In the subsequent lowtemperature experiments, Doppler broadening of the positron annihilation $\gamma$-ray spectrum at each temperature was recorded. To avoid any effects of hysteresis, ${ }^{24}$ the sample was first cooled slowly to the lowest temperature, allowed to stabilize there for several hours and the measurements were then carried out after raising the temperature to the desired value. Two series of such measurements were performed (to check the reproducibility of the results) and the entire experiment was then repeated on a reference polyacrylamide polymer sample.

The $S$ parameter vs temperature data for the silverpolymer nanocomposite is shown in Fig. 7(b). While the overall behavior of a decreasing trend in the $S$ parameter curve with decreasing temperature is in accordance with the effects of bulk contraction of the solid, certain additional features are observed around $80 \mathrm{~K}$. There is a sudden sharp further decrease in $S$ followed by a rise and then the curve extends to lower temperatures as if it were the extrapolation of the original decreasing trend. This anomalous fall and rise can probably be an indication of the occurrence of a change in the material characteristics leading to a phase transition, but shadowed by other possible effects of the polymer medium. The data on the pure reference polymer are shown in Fig. 7(a). Here the effects of contraction are unambiguous, even though the hump appearing in the region $50-150 \mathrm{~K}$ is not properly understood. Nevertheless, a vivid indication of 


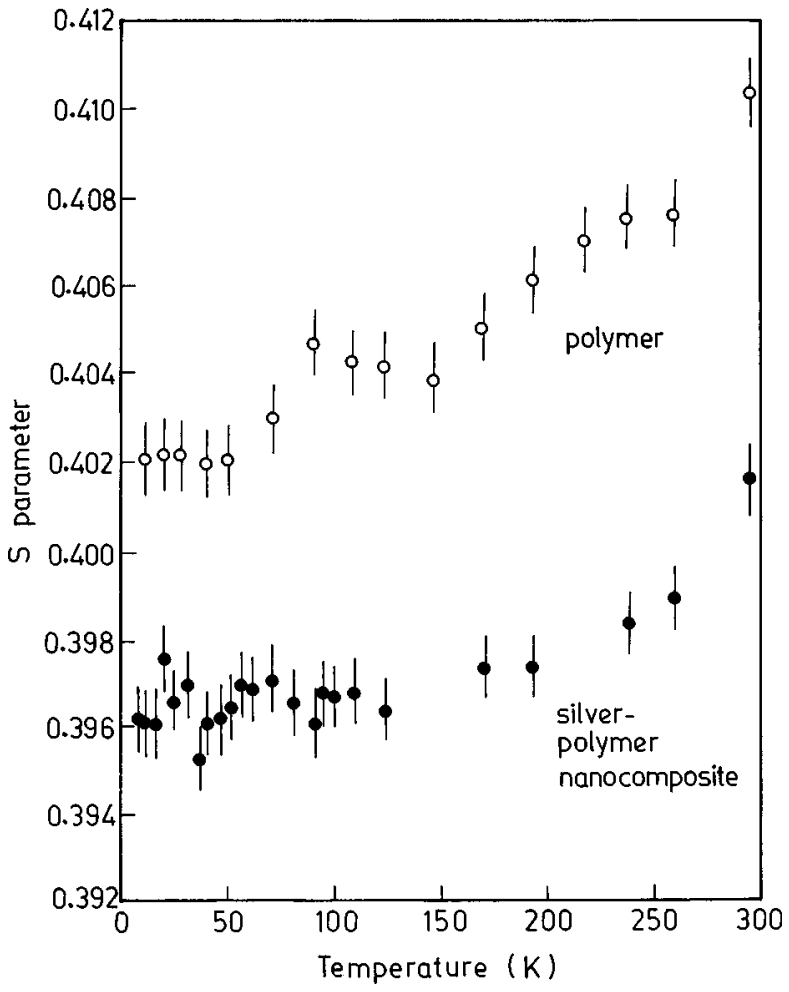

FIG. 7. The variation of the $S$ parameter with sample temperature for (a) the pure reference polymer and (b) the silver-polymer nanocomposite.

certain special features pertaining to the silver nanoparticles can be emphasized from a comparison of Figs. 7(a) and 7(b), especially at temperatures below $100 \mathrm{~K}$.

In order to get additional insight into the minute aspects of these changes, the data on the silver-polymer nanocomposite were analyzed further using a mixture rule ${ }^{25}$ to decompose the measured $S$ parameter $\left(S_{c}\right)$ into the respective contributions from the nanocrystalline metal particles $\left(S_{m}\right)$ and from the polymer medium $\left(S_{p}\right)$. Here we have assumed that the two phases present contribute independently to the Doppler broadening. For this, the volume fraction $v$ of the silver nanoparticles in the nanocomposite was estimated from the measured densities of the nanocomposite and the pure polymer. Using the relation

$$
S_{c}=S_{m} v+S_{p}(1-v),
$$

the values of $S_{m}$ at various temperatures were calculated.

The results of the above analysis are shown in Fig. 8(a). Looking from the direction of decreasing temperature, a twostep rise in the estimated fractional contribution $S_{m}$ is observed. The first rise seems to begin at $210 \mathrm{~K}$ and then levels off in between 150 to $80 \mathrm{~K}$. A further sharp rise starting from $80 \mathrm{~K}$ and flattening off at $60 \mathrm{~K}$ is significant in the context of a possible transition at this stage, as can be predicted from the work of Wood and Ashcroft. ${ }^{9}$ According to these authors, the average one-electron energy-level separation for a metal nanoparticle can be written as

$$
\Delta=E_{f} / N,
$$

where $E_{f}$ is the Fermi energy for metal concerned and $N$ is the number of atoms in the particle. Assuming that the

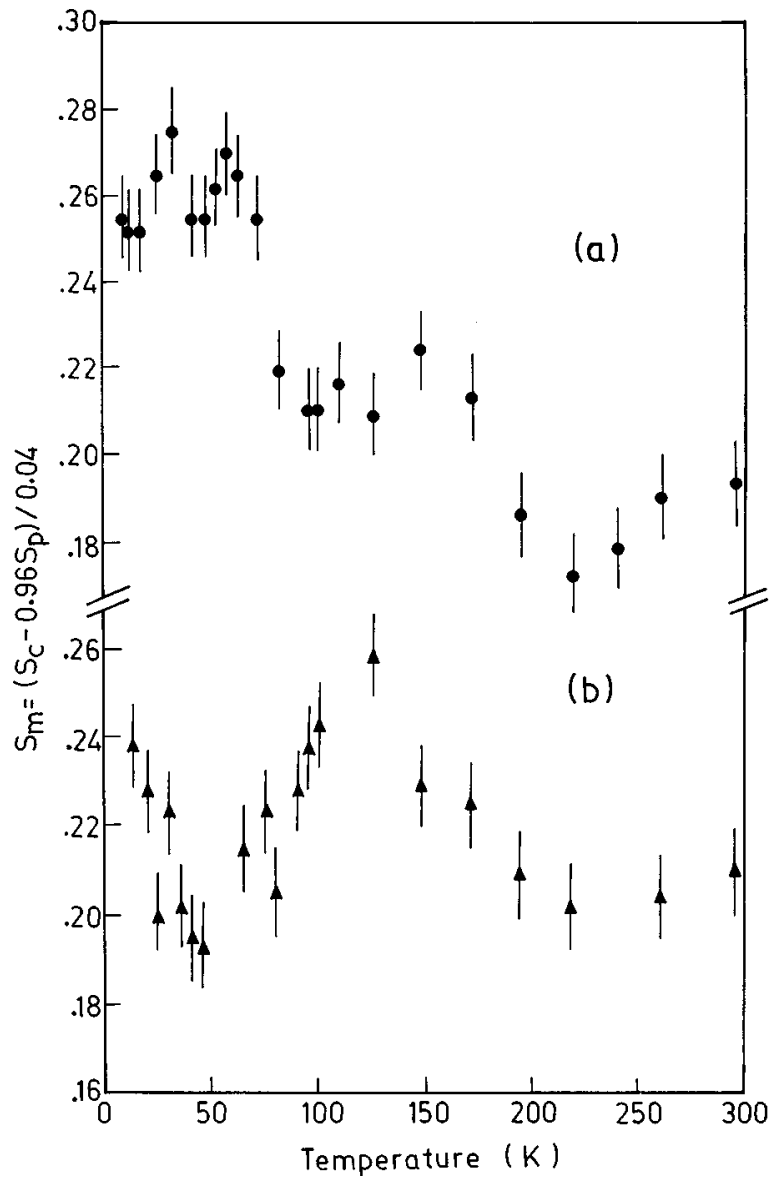

FIG. 8. The decomposed fractional parameter $S_{m}$ vs sample temperature in the first series (circles) and in the second series (triangles), showing the metal-to-semiconductorlike transition and the shift of transition due to agglomeration of silver nanoparticles.

nanocrystalline silver particles have got the same fcc structure as that of bulk metallic silver, where the unit-cell volume of $a^{3}$ on the average consists of 4 atoms, a nanoparticle of radius $r$ will consist of $N=16 \pi r^{3} / 3 a^{3}$ atoms. Substituting this number in Eq. (3) and using $E_{f}=5.5 \mathrm{eV}$ (equivalent to $64000 \mathrm{~K}$ ) for silver, the sizes of particles contributing to the rise at 210 and $80 \mathrm{~K}$ are, respectively, found to be 2.1 and $3.0 \mathrm{~nm}$. The histogram of silver nanoparticle-size distribution in the nanocomposite [Fig. 6(a)] seems to support this interpretation, as a significant number of nanoparticles of the sizes mentioned above are observed in the sample. It may be noted that majority of the particles (of sizes larger than 5.4 $\mathrm{nm}$ ) do not exhibit this transition up to the lowest temperature of $13 \mathrm{~K}$ that could be attained in the cryogenerator. Also, while the presence of nanoparticles of diameter $3.0 \mathrm{~nm}$ is beyond ambiguity [Fig. 6(a)], that of an extremely low diameter of $2.1 \mathrm{~nm}$ is not visibly evident from the TEM data. The increase in the value of the $S$ parameter is ascribed to a metal-to-semiconductorlike transition. The $S$ parameter by definition is a measure of the relative number of lowmomentum electrons annihilated by positrons. ${ }^{26}$ Due to an increased energy-level spacing as in a semiconductor, the overlap of the wave function of the relatively highmomentum core electrons with that of the thermally diffusing positron will become less significant. As a result, the positron annihilation $\gamma$-ray spectra will be dominated by 
TABLE VI. Positron lifetimes and intensities in the two samples used in the low-temperature experiments before and after the experiments.

(a) Pure reference polymer

\begin{tabular}{|c|c|c|c|c|c|c|c|}
\hline & VOF & $\tau_{1}(\mathrm{ps})$ & $\tau_{2}(\mathrm{ps})$ & $\tau_{3}(\mathrm{ps})$ & $I_{1}(\%)$ & $I_{2}(\%)$ & $I_{3}(\%)$ \\
\hline Before & 1.340 & 160 & 402 & 1587 & 30.6 & 47.5 & 21.9 \\
\hline After & 1.104 & 190 & 419 & 1588 & 34.8 & 43.6 & 21.6 \\
\hline \multicolumn{8}{|c|}{ (b)Silver-polymer nanocomposite } \\
\hline Before & 1.212 & 184 & 391 & 1338 & 37.8 & 49.5 & 12.7 \\
\hline After & 1.125 & 199 & 414 & 1386 & 45.4 & 43.3 & 11.3 \\
\hline
\end{tabular}

events from the low-momentum valence or conduction electrons, causing the sharpening of the spectral line shape around the peak or, in other words, an increase in the value of the $S$ parameter. The interpretation of a transition at $80 \mathrm{~K}$ is also supported by earlier observations, ${ }^{24}$ which had observed an anomalous change of electrical resistance at $80 \mathrm{~K}$ in the case of silver nanoparticles of sizes approximately 2.9 $\mathrm{nm}$ grown in an ion-exchanged glass ceramic and attributed the same to a transition from a metallic to a semiconductorlike behaviour.

Surprisingly, on a repetition of the whole experiment on the same samples, the transition earlier observed at $80 \mathrm{~K}$ was found shifted to $45 \mathrm{~K}$ [Fig. 8(b)]. Going by the WoodAshcroft theory of divergence of the energy-level spacing in very small particles, ${ }^{9}$ the shift in the temperature of transition seems to indicate an agglomeration of the silver nanoparticles. Figure 6(b) gives the histogram of silver particles in the nanocomposite after the low-temperature positron annihilation experiment. The average particle diameter is seen to have increased to $14.3 \mathrm{~nm}$. The first characteristic steep rise of $S_{m}$ at $210 \mathrm{~K}$ however did not get shifted. This leads to the conclusion that the anomaly at this temperature may not be related to a transition of the type discussed as above. That the silver nanoparticles can agglomerate, resulting in a lowering of the transition temperature, as expected from Eq. (3), can be explained based on the effects of positron irradiation of the bulk polymer, as explained in further discussions.

Table VI presents the results of positron lifetime measurements on the silver-polymer nanocomposite and the pure reference polymer, both before and after the low-temperature experiments. As illustrated in the table, each positron lifetime spectrum has been resolved into three lifetime components $\tau_{1}, \tau_{2}$, and $\tau_{3}$, with relative intensities $I_{1}, I_{2}$, and $I_{3}$. Using the scheme of analysis outlined in Ref. 15, we have estimated the positron lifetime in the bulk polymer to be 171 ps before the experiments and 207 ps after the experiments. Again, the increase in the lifetime after the experiments is interpreted in terms of positron irradiation effects, to be discussed afterwards. The longer two positron lifetime components have the same meaning as in Sec. III B but are not relevant in the present discussion.

Similar changes appear to have taken place in the silverpolymer nanocomposite also (Table VI). Here $\tau_{1}$ further incorporates the lifetime of positrons annihilating at the diffuse vacancy clusters on the nanocrystalline silver grain interfaces. As already pointed out, the annihilation of positrons captured by the nanoparticles occurs only at the grain surfaces, as the thermalized positrons diffuse out on to the surfaces of the grains that are smaller in size compared to the mean diffusion length of positrons. Using the extended scheme of analysis presented in Ref. 15, we have been able to calculate the positron lifetime at the interfacial defects as 238 ps before the low-temperature experiments and 203 ps after the experiments. Following the established observations, the reduction of positron lifetime at the grain interfaces is directly correlated with an increase in the grain size. ${ }^{20}$ This has been attributed to the net reduction in the interfacial defect volume due to the shrinkage of the defects when the grain surface expands. Thus the change of positron lifetime from 238 to 203 ps is clearly indicative of the formation of larger grains due to the agglomeration of smaller nanoparticles. This is consistent with the shift of the transition temperature from 80 to $45 \mathrm{~K}$ in the second series of measurements, as discussed earlier. Having thus accounted for the observed shift in the transition temperature, it remains rather puzzling as to why a similar shift did not occur for the transition at $210 \mathrm{~K}$. Perhaps the rise in $S_{m}$ at this temperature is not related to the kind of metal-to-semiconductorlike transition under discussion and the source of this anomaly may altogether be of a different nature. That the particle size of $2.1 \mathrm{~nm}$ corresponding to this temperature in accordance with Eq. (3) is rather very small, not very much in consistence with the TEM histogram in Fig. 6.(b), further strengthens this apprehension.

It is now worth trying to understand the mechanism leading to the agglomeration of the nanoparticles during the experiments. Over a period of about $330 \mathrm{~h}$ during which the total measurements on each sample were carried out, the sample had been continuously bombarded by positrons (of end-point energy $\sim 540 \mathrm{keV}$ ) and $\gamma$ rays (both 1.28 and $0.511 \mathrm{MeV}$ ) from the ${ }^{22} \mathrm{Na}$ source. Considering that the source had a strength of approximately $10 \mu \mathrm{Ci}$, giving 3.7 $\times 10^{5}$ disintegrations per second, and that the source was deposited over an active area of diameter $2 \mathrm{~mm}$, the energy deposited in the sample during the first series of measurements $(\sim 165 \mathrm{~h})$ was about $8.75 \mathrm{Mrad}^{11}$ (It may be mentioned here for clarification that, for the experiments discussed in the previous sections, a weaker source of strength $\sim 1 \mu \mathrm{Ci}$ had been used and a given set of samples was sandwiched with the source for not more than $16 \mathrm{~h}$. The positron and $\gamma$ irradiation effects were therefore not considered to be relevant.) This could in fact lead to the breaking of the polymer chains whereby additional free space would become available for the trapping of positrons. That the positron lifetime in the bulk polymer increased from 171 to 207 ps during the completion of the experiments bears proof to this argument. The additional free space made available by the radiation-induced damage on the polymer chains also pro- 
vides a favorable environment for the nanoparticles to migrate and agglomerate, leading to an effective increase in their sizes. As the size of the nanoparticles increases, the atomic arrangement on the surface becomes less fragile, leading to a partial reduction of the sizes of the vacancy clusters on the surface. The positrons trapped in these vacancy clusters of reduced size will then exhibit a shorter lifetime, as observed in the final measurements.

\section{SUMMARY AND CONCLUSIONS}

We have presented here the results of positron lifetime and Doppler broadening measurements on samples of polyacrylamide polymer in which silver nanoparticles were chemically grown and characterized. (i) We observed that, as in the case of positron annihilation experiments on nanoparticles grown by the conventional methods, positrons can get trapped into the diffuse vacancy clusters on the grain surface and the nature and size of these clusters can be quantitatively understood from the measured positron lifetime. The present measurements indicated vacancy clusters of sizes corresponding to 3-4 monovacancies on the grain surfaces. (ii) It is further pointed out that the open spaces between the grain surface and the surrounding innermost polymer layer can also trap positrons, providing them with a characteristically long lifetime. Molecular-dynamic simulation studies can throw more light on this concept, which is at least qualitatively identical to a solid bubble in a polymer matrix. (iii)

*Present address: Instituto de Estructura de la Materia, Consejo Superior de Investigaciones Cientificas, Serrano 123, 28006 Madrid, Spain. Electronic address: mukherje@foton $\varnothing . i e m$. csic.es ${ }^{\dagger}$ Also affiliated with Jawaharlal Nehru Centre for Advanced Scientific Research, Bangalore 560 064, India. Electronic address: mls@iacs.ernet.in

†Electronic address: gopal@anp.saha.ernet.in

${ }^{1}$ R. Birringer, H. Gleiter, H. P. Klein, and P. Marquardt, Phys. Lett. 102A, 365 (1984).

${ }^{2}$ Hans-Eckhardt Schaefer, in Mechanical Properties and Deformation Behavior of Materials Having Ultra-Fine Microstructures, edited by M. Nastasi et al. (Kluwer-Academic, The Netherlands, 1993), p. 81.

${ }^{3}$ J. R. Poulsen, A. Horsewell, M. Eldrup, E. Johnson, and A. Johansen, J. Phys. Condens. Matter 6, 5397 (1994).

${ }^{4}$ H. E. Schaefer, and R. Wurschum, Phys. Lett. A 119, 370 (1987).

${ }^{5}$ H. E. Schaefer, R. Wurschum, R. Birringer, and H. Gleiter, Phys. Rev. B 38, 9545 (1988).

${ }^{6}$ Tokushi Kizuka, Yuko Nakagami, Tohru Ohata, Ikuzo Kanazawa, Hideki Ichinose, Hideoki Murakami, and Yoichi Ishida, Philos. Mag. A 69, 551 (1994).

${ }^{7}$ X. Y. Qin, J. S. Zhu, X. Y. Zhou, and X. J. Wu, Phys. Lett. A 193, 335 (1994).

${ }^{8}$ S. T. Lee, G. Apai, M. G. Mason, R. Benbow, and Z. Hurych, Phys. Rev. B 23, 505 (1981).

${ }^{9}$ D. M. Wood and N. W. Ashcroft, Phys. Rev. B 25, 6255 (1982).

${ }^{10}$ H. P. Klug and L. E. Alexander, X-ray Diffraction Procedures for Polycrystalline and Amorphous Materials, 2nd ed. (WileyInterscience, New York, 1974), p. 689.

${ }^{11}$ Takenori Suzuki, Taichi Miura, Yuichi Oki, Masaharu Numajiri, Kenjiro Kondo, and Yasuo Ito, Radiat. Phys. Chem. 45, 657 (1995).
The o-Ps lifetime $\tau_{3}$ essentially reflected the properties of the free-volume defects in the polymer. During the isochronal annealing, the polymer expanded by shrinking these defects and the resultant decrease of the o-Ps lifetime is clearly reflected. (iv) The lifetime of positrons trapped at the grain surface defects and the grain-polymer interface decreased with increasing grain size, in agreement with the results reported by others in the literature. (v) The isothermal annealing at $473 \mathrm{~K}$ resulted in the interdiffusion of the surface atoms on the nanoparticles and the polymer atoms. (vi) It is shown that the metal-to-semiconductorlike phase transition, predicted in the case of nanosized metal particles and also seen recently in the electrical resistivity measurements, can be investigated using positrons as alternative experimental probes. In the present investigation on metal-polymer nanocomposites containing nanosized silver particles, this transition has been indicated as a rise in the decomposed $S$ parameter at around $80 \mathrm{~K}$. The transition temperature is observed to be rather sensitive to the average particle size, as expected from established theoretical predictions.

\section{ACKNOWLEDGMENTS}

We thank Professor Prasanta Sen for moral support and encouragement. M. Mukherjee and D. Chakravorty acknowledge the support from a foreign research Grant No. N0001493-1-0040 by the Office of Naval Research, Virginia, U.S.A.

${ }^{12}$ R. A. Naslud, Ph. L. Jones, and A. Crowson, Mater. Sci. Forum 175-178, 739 (1995).

${ }^{13}$ P. Kirkegaard, M. Eldrup, O. E. Mogensen, and N. J. Pedersen, Comput. Phys. Commun. 23, 307 (1981).

${ }^{14}$ B. Roy and D. Chakravorty, J. Phys. Condens. Matter 2, 9323 (1990).

${ }^{15}$ A. Uedono, R. Sadamoto, T. Kawano, S. Tanigawa, and T. Uryu, J. Polym. Sci. B: Polym. Phys. 33, 891 (1995).

${ }^{16}$ H. Nakanishi, S. J. Wang, and Y. C. Jean, in Positron Annihilation Studies of Fluids, edited by S. C. Sharma (World Scientific, Singapore, 1988), p. 292.

${ }^{17}$ Y. C. Jean, Nucl. Instrum. Methods Phys. Res. B 56/57, 615 (1991).

${ }^{18}$ J. Liu, Q. Deng, and Y. C. Jean, Macromolecules 26, 7149 (1993).

${ }^{19}$ E. Soininen, H. Huomo, P. A. Hattunen, J. Mukinen, A. Vehanen, and P. Hautojarvi, Phys. Rev. B 41, 6227 (1990).

${ }^{20}$ H. Y. Tong, B. Z. Ding, J. T. Wang, K. Lu, J. Jiang, and J. Zhu, J. Appl. Phys. 72, 5124 (1992).

${ }^{21}$ R. Dittmar, R. Wurschum, W. Ulfert, and H. E. Schaefer, in Proceedings of the Eleventh International Conference on Positron Annihilation, Missouri, 1997 [Mater. Sci. Forum (to be published)].

${ }^{22}$ K. O. Jensen, in Fundamental Aspects of Inert Gases in Solids, edited by S. E. Donnelly and J. H. Evans (Plenum, New York, 1991), p. 195.

${ }^{23}$ K. O. Jensen and R. M. Nieminen, Phys. Rev. B 35, 2087 (1987); 36, 8219 (1987).

${ }^{24}$ B. Roy and D. Chakravorty, Solid State Commun. 87, 71 (1993).

${ }^{25}$ W. D. Kingery, Introduction to Ceramics (Wiley, New York, 1967), p. 719.

${ }^{26}$ R. W. Siegel, Annu. Rev. Mater. Sci. 10, 393 (1980). 